Series A

I. MATHEMATICA

426

\title{
RAUMWINKEL UND QUASIKONFORMITÄT
}

VON

OSSI TAARI

H E L S I N K I 1968

S U O M A L A I N E T I E DEAKATEM I A 
Am 9 April 1968 vorgelegt von K. I. Virtanen und Lauri Myrberg 


\section{Einleitung}

Die Frage nach der Charakterisierung einer quasikonformen Abbildung mit Hilfe der Winkelverzerrung ist früher im zweidimensionalen Fall von Agard-Gehring [2] und vom Verfasser [8] untersucht worden. In der vorliegenden Arbeit stellen wir uns die Aufgabe, eine ähnliche Definition im $n$-dimensionalen Raum anzugeben. Wie in dem Falle $n=2$, ist es auch hier das erste Problem, einen geeigneten Winkelbegriff einzuführen. Zu diesem Zweck wird das Verhalten eines $n$-Kegels bei einem Homöomorphismus untersucht und ein verallgemeinerter Raumwinkel definiert mit Hilfe gewisser Kugeln, die im Innern der Bildfläche liegen.

Geeignete Beschränkungen dieser Bildwinkel garantieren die Quasikonformität des betreffenden Homöomorphismus, wie in $\S 3$ bewiesen wird. Für die absolute Stetigkeit auf Geraden und die fast überall bestehende Differenzierbarkeit ist es hierbei genügend, nur einander ähnliche Kegel zu betrachten, deren Achsen parallel zu den Koordinatenachsen liegen. Umgekehrt wird in $\S 4$ die Winkelverzerrung bei einer quasikonformen Abbildung untersucht.

Bei der Auserbeitung dieser Abhandlung habe ich ein Manuskript von Agard [1] eingesehen, in dem die Quasikonformität mit Hilfe des Winkelbegriffes von Agard-Gehring (vgl. [2]) charakterisiert wird. Wir werden in $\S 5$ den Zusammenhang dieses Winkelbegriffes mit unserem näher untersuchen. Ferner hat Caraman [3] mitgeteilt, dass er und Corduneanu [4] dasselbe Problem gelöst haben.

\section{§ 1. Quasikonformität in dem Raume $\mathbf{R}^{n}$}

Es sei $\left(e^{1}, \ldots, e^{n}\right)$ eine fixierte Basis von $R^{n}$. Jeder Punkt $x \in R^{n}$ hat dann die eideutige Koordinatendarstellung

$$
x=\sum_{i=1}^{n} x_{i} e^{i}
$$

und der Winkel $(x, y)$ zwischen zwei von Null verschiedenen Vektoren $x$ und $y$ lässt sich aus der Formel 


$$
\cos (x, y)=\frac{(x \mid y)}{|x||y|}, \quad 0 \leqq(x, y) \leqq \pi
$$

berechnen, worin $(x \mid y)$ das Skalarprodukt $\sum_{i=1}^{n} x_{i} y_{i}$ ist.

Die Definition der Quasikonformität in $R^{n}$ wird gewöhnlich mit Hilfe des Moduls einer Bogenfamilie gegeben. Zu diesem Zweck betrachte man eine Familie $\Gamma$ der Jordanbogen. Wir bezeichnen mit $F(T)$ eine Menge der Funktionen $\varrho$ mit den Eigenschaften:

$1^{\circ} . \varrho(x) \geqq 0$ für jedes $x \in R^{n}$,

$2^{\circ}$. $\varrho$ ist Borel-messbar,

$3^{\circ} . \int_{\gamma} \varrho d s \geqq 1$ für jedes $\gamma \in \Gamma$.

Man setzt

$$
M(\Gamma)=\inf _{\varrho \in F(\Gamma)} \int_{R^{n}} \varrho^{n} .
$$

Die Zahl $M(\Gamma)$ heisst der Modul von $T$. Eine quasikonforme Abbildung wird nun auf folgende Weise erklärt.

Definition. Ein Homöomorphismus $f$ von $G \subset R^{n}$ ist $K$-quasikonform, $1 \leqq K<\infty$, wenn die Doppelungleichung

$$
\frac{1}{K} M(\Gamma) \leqq M(f(\Gamma)) \leqq K M(\Gamma)
$$

für jede Bogenfamilie $\Gamma$ in $G$ gilt.

Eine mit dem Obigen äquivalente Charakterisierung der Quasikonformität ist die sogenannte analytische Definition, die auf dem Begriff der "absoluten Stetigkeit auf Geraden» beruht. Eine stetige Abbildung $f: G \rightarrow G^{\prime}$ ist absolut stetig auf Geraden oder $A S G$ in $G$, wenn sie für jedes Intervall $I=\left\{x \in R^{n} \mid a_{i}<x_{i}<b_{i}, \quad i=1,2, \ldots, n\right\}, \quad \bar{I} \subset G$, absolut stetig auf fast allen achsenparallelen Strecken ist, die in $\bar{I}$ liegen. Dies bedeutet, dass diejenige Teilmenge $E$ von $I_{i}=\left\{x \in \bar{I} \mid x_{i}=a_{i}\right\}$, für die $f$ nicht absolut stetig auf der Strecke $\left\{y+t e^{i} \mid y \in E, 0 \leqq t \leqq b_{i}-a_{i}\right\}$ ist, eine Nullmenge in bezug auf das $(n-1)$-dimensionale Lebesguesche Mass ist. Eine in $G A S G$-Funktion $f$ besitzt endliche partielle Ableitungen für fast alle $x \in G$. Sind die partiellen Ableitungen von $f$ dazu lokal $L^{n}$ integrierbar, so sagen wir, dass $f$ eine $A S G_{n}$-Funktion ist. Hieraus folgt, 
dass $f$ für fast alle $x \in G$ differenzierbar ist (vgl. Väisälä [10], Lemma 3), und wir bezeichnen hierbei

$$
L(x, f)=\max _{|h|=1}|D f(x) h|, \quad l(x, f)=\min _{|h|=1}|D f(x) h|,
$$

wo $D f(x)$ die Ableitung von $f$ im Punkte $x$ ist. Ferner sei $J(x, f)$ die Jacobische Funktionaldeterminante von $f$.

Die analytische Definition der Quasikonformität lautet (vgl. Väisälä [10], Theorem 2): Ein Homöomorphismus $f: G \rightarrow G^{\prime}$ ist $K$-quasikonform genau dann, wenn er $A S G_{n}$ in $G$ ist und für fast jedes $x \in G$ die Doppelungleichung

$$
(L(x, f))^{n} / K \leqq|J(x, f)| \leqq K(l(x, f))^{n}
$$

\section{befriedigt.}

In den nachstehenden Betrachtungen wird das Hausdorffsche lineare Mass angewandt, das für die Mengen von $R^{n}$ auf folgende Weise erklärt wird: Es sei $E$ eine Menge in $R^{n}$. Zu jedem $\delta>0$ betrachte man alle abzählbaren Überdeckungen $\mathfrak{B}$ von $E$ mit den Punktmengen $B$, von denen jede einen Durchmesser $d(B)<\delta$ besitzt. Man setze

$$
\Lambda(E, \delta)=\inf \Sigma d(B)
$$

und definiere das äussere lineare Mass von $E$ als den Grenzwert

$$
A^{*}(E)=\lim _{\delta \rightarrow 0} A(E, \delta) .
$$

Auf einer Geraden stimmt $\Lambda^{*}(E)$ mit dem Lebesgueschen äusseren Längenmass überein. Ist $E A^{*}$-messbar, so bezeichnet man $\Lambda^{*}(E)$ mit $\Lambda(E)$. Ferner ist $\Lambda^{*}$ ein reguläres Carathéodory-Mass, wonach alle Borelschen Mengen $\Lambda^{*}$-messbar sind.

\section{§ 2. Definition eines Raumwinkels}

Es sei $f: G \rightarrow G^{\prime}$ ein Homöomorphismus zwischen den Gebieten $G$ und $G^{\prime}$ in $R^{n}$ und $\alpha$ ein fester Winkel, $0<\alpha<\pi / 2$. Die Menge aller Punkte $x \in G$ der Doppelkegelfläche $\left|\cos \left(x-x^{0}, v\right)\right|=\cos \alpha$, wo $x^{0}$ ein Punkt von $G$ und $v,|v|=1$, ein fester Vektor ist, bezeichnen wir mit $W\left(x^{0}, v, \alpha\right)$. Mit Hilfe der Bildmenge $W^{\prime}\left(x^{0}, v, \alpha\right)=f\left(W\left(x^{0}, v, \alpha\right)\right)$ wollen wir das $f$-Bild des Kegelwinkels a auf geeignete Weise definieren. $\mathrm{Zu}$ diesem Zweck betrachten wir für ein reelles $t \neq 0$ die Kugel $K_{r}(t)$ mit dem Mittelpunkt in $f\left(x^{0}+t v\right)$ und mit dem Radius $r=r\left(x^{0}, x^{0}+t v\right)$, wo $r\left(x^{0}, x^{0}+t v\right)$ die Entfernung des Punktes $f\left(x^{0}+t v\right)$ von der Menge $W^{\prime}\left(x^{0}, v, \alpha\right)$ ist. 
Man setze für $m=1,2, \ldots$

$$
\omega_{m}\left(x^{0}, v, \alpha\right)=\inf _{0<|t|<\frac{1}{m}} \frac{r\left(x^{0}, x^{0}+t v\right)}{\left|f\left(x^{0}+t v\right)-f\left(x^{0}\right)\right|} .
$$

Dann ist $\left(\omega_{m}\right)$ eine nicht abnehmende Folge, und wir definieren den Bildwinkel des erzeugenden Winkels von $W\left(x^{0}, v, \alpha\right)$ durch den Ausdruck

$$
\arcsin \omega\left(x^{0}, v, \alpha\right) \text {, }
$$

in dem

$$
\omega\left(x^{0}, v, \alpha\right)=\lim _{m \rightarrow \infty} \omega_{m}\left(x^{0}, v, \alpha\right)
$$

ist, $\quad 0 \leqq \omega\left(x^{0}, v, \alpha\right) \leqq 1$. Bei der Definition dieses Winkels genügt es natürlich, sich auf eine beliebige Umgebung von $x^{0}$ zu beschränken. Ferner sieht man unmittelbar, dass arc $\sin \omega\left(x^{0}, v, \alpha\right)$ dieselbe Bedeutung wie $\alpha$ hat, falls $W^{\prime}\left(x^{0}, v, \alpha\right)$ eine gewöhnliche Kegelfläche ist, deren erzeugender Winkel kleiner als $\pi / 2$ ist.

\section{§ 3. Definition der Quasikonformität mit Hilfe der Raumwinkel}

Wir beweisen folgendes ${ }^{1}$ :

Satz 1. Es sei $f: G \rightarrow G^{\prime}$ ein Homöomorphismus zwischen den Gebieten $G$ und $G^{\prime}$ in $R^{n}$. Gelten die Bedingungen

$1^{\circ} . \operatorname{arc} \sin \omega\left(x, e^{i}, \alpha\right)>0, i=1,2, \ldots, n$, für jedes $x \in G$,

$2^{\circ}$. arc $\sin \omega(x, v, \alpha) \geqq \alpha / K$ für jedes $v,|v|=1$, unf für fast alle $x \in G$,

wobei $K \geqq 1$ eine endliche Konstante ist, für jedes $\alpha, 0<x<\pi / 2$, so ist $f K^{n-1}$-quasikonform. Die Zahl $K^{n-1}$ ist hierbei die bestmögliche.

Der Beweis dieses Satzes wird in den folgenden drei Hilfssätzen auf Grund der analytischen Definition geführt. In Lemma 1 und Lemma 2 wird die $A S G_{n}$-Eigenschaft von $f$ bewiesen, und hierfür braucht man nur eine Klasse von einander ähnlichen Kegeln zu betrachten, deren Achsen parallel zu den Koordinatenachsen liegen. Der Kürze halber werden die Bedingungen $1^{\circ}$ und $2^{\circ}$ in Lemma 1 und 2 für (') formuliert, was natürlich keine Beschränkung bedeutet.

1) Wie aus dem Beweis leicht hervorgeht, gilt Satz 1 auch dann, wenn der Ausdruck $\operatorname{arc} \sin \omega$ in den Bedingungen $1^{\circ}$ und $2^{\circ}$ durch $\omega$ ersetzt wird. 
Lemma 1. Gilt für einen festen Winkel $\alpha, 0<\alpha<\pi / 2$,

$1^{\circ}$. $\omega\left(x, e^{i}, \alpha\right)>0$ für jedes $x \in G$,

$2^{\circ}$. $\omega\left(x, e^{i}, \alpha\right) \geqq \alpha / K$ für fast alle $x \in G$,

$i=1,2, \ldots, n$, so ist $f A S G$ in $G$.

Beweis. Wir wählen ein $n$-Intervall $I=\left\{x \in R^{n} \mid a_{i}<x_{i}<b_{i}\right\}, \quad \bar{I} \subset G$, und bezeichnen seine Projektion auf der Ebene $x_{1}=0$ mit $I_{0}$. Für jede Borelsche Menge $E \subset I_{0}$ sei $Q_{E}=\left\{x+t e^{1} \mid x \in E, \quad a_{1}<t<b_{1}\right\}$. Das Lebesguesche Volumenmass $F(E)=m\left(f\left(Q_{E}\right)\right)$ ist eine nichtnegative additive Mengenfunktion und besitzt daher eine endliche Ableitung für fast alle $z \in I_{0}$. Wegen der Stetigkeit von $f$ ist $\omega_{m}, m=1,2, \ldots$, und also auch $\omega$ messbar in $G$, und nach dem Satz von Fubini gilt daher die Abschätzung

$$
\omega\left(x, e^{1}, \alpha\right) \geqq \alpha / K
$$

fast überall auf der Strecke $Q_{z}$ für fast jedes $z \in I_{0}$. Wir wählen einen solchen Punkt $z \in I_{0}$, für den diese zwei Bedingungen erfüllt sind, und bezeichnen die entsprechende Strecke $Q_{z}$ mit $J$. Wegen der Symmetrie wird die ASG-Eigenschaft von $f$ bewiesen, wenn wir zeigen, dass $f$ absolut stetig auf $J$ ist; der Einfachheit halber nehmen wir an, dass $J$ die Strecke $\left\{x \in R^{n} \mid 0<x_{1}<1, x_{2}=\ldots=x_{n}=0\right\}$ ist. Ferner bezeichne man mit $\sigma(0)$ den Grenzwert $\lim m\left(Z^{\prime}(\varrho)\right) / m(Z(\varrho))$, wobei $Z(\varrho)$ der Zylinder $\left\{x \in R^{n} \mid 0<x_{1}<1, \quad \stackrel{\varrho \rightarrow 0}{x_{2}^{2}}+\ldots+x_{n}^{2}<\varrho^{2}\right\}$ ist. In den nachstehenden Betrachtungen wird das $f$-Bild einer Menge $A$ allgemein mit $A^{\prime}$ bezeichnet.

Es sei $E \subset J$ eine beliebige in bezug auf das lineare Mass messbare Menge. Für jedes $s>0$ bezeichnen wir mit $H_{m s}$ die Menge derjenigen Punkte $x$ von $J$, für die $\omega_{m}\left(x, e^{\mathbf{1}}, \alpha\right) \geqq \alpha / s$ ist. Wegen der Stetigkeit von $f$ sind $H_{m s}$ und $H_{m s}^{\prime}$ abgeschlossen. Die Borelsche Menge $H_{s}=\bigcup_{m=1}^{\infty} H_{m s}=$ $\lim H_{m s}$ enthält alle Punkte von $J$, für die $\omega\left(x, e^{1}, x\right)>x / s$ ist. $m \rightarrow \infty$

Wir wollen zunächst die Gültigkeit der Abschätzung

$$
A^{*}\left(E^{\prime} \cap H_{s}^{\prime}\right) \leqq \varkappa \frac{s}{\alpha} \sqrt[n]{\sigma(0)(\Lambda(E))^{n-1}}
$$

für jedes $s>0$ beweisen, wo $x$ die Konstante $\sqrt[n]{2 \tan ^{n-1} \alpha \Omega_{n-1} / \Omega_{n}}$ ist ist $^{1}$. Nach der Gleichung

$$
\Lambda\left(H_{s}^{\prime}\right)=\lim _{m \rightarrow \infty} \Lambda\left(H_{m s}^{\prime}\right)
$$

$\left.{ }^{1}\right)$ Wie gewöhnlich, bezeichnet $\Omega_{j}$ das Mass des Einheitskugels $|x|<1$ in $R i$. 
genügt es hierfür, die Beziehung

$$
\Lambda^{*}\left(E^{\prime} \cap H_{m s}^{\prime}\right) \leqq \varkappa \frac{s}{\alpha} \sqrt[n]{\sigma(0)(\Lambda(E))^{n-1}}
$$

für jedes ganzzahlige $m$ zu begründen.

$\mathrm{Zu}$ diesem Zweck überdecke man für festes $m$ die Menge $E \cap H_{m s}$ mit offenen punktfremden Intervallen $O_{i} \subset J, i=1,2, \ldots$. Die Vereinigungsmenge $O=\bigcup_{i=1}^{\infty} O_{i}$ befriedigt dann die Beziehung

$$
E \cap H_{m s} \subset O \cap H_{m s} \subset O \text {. }
$$

Wir wählen eine beliebige ganze Zahl $M>0$ und schätzen die Summe $\sum_{i=1}^{M} \Lambda\left(O_{i}^{\prime} \cap H_{m s}^{\prime}\right)$ nach oben ab.

Man zerlege das Intervall $\bar{J}$ in $p, p \geqq m$, gleich grosse abgeschlossene Teilintervalle und wähle unter diesen diejenigen, die gemeinsame Punkte mit $\bigcup_{i=1}^{M} O_{i}$ besitzen. Bezeichnet man diese Intervalle mit $J_{k}, k=$ $1,2, \ldots, N$, so gilt $\bigcup_{1} O_{i} \subset \bigcup_{1} J_{k}$. Da die Gesamtlänge derjenigen Teilintervalle von $\bigcup_{1}^{N} J_{k}$, die $O_{i}$ überdeckt, kleiner als $\Lambda\left(O_{i}\right)+\frac{2}{p}$ ist, gilt die Ungleichung

$$
N \cdot \frac{1}{p}=\sum_{k=1}^{N} \Lambda\left(J_{k}\right)<\sum_{i=1}^{M} \Lambda\left(O_{i}\right)+M \cdot \frac{2}{p} .
$$

Um die gewünschte Abschätzung zu gewinnen, schätzen wir zunächst den Durchmesser $d_{k}$ von $J_{k}^{\prime} \cap H_{m s}^{\prime}$ nach oben ab. Sind $a$ und $b$ zwei Punkte von $J_{k} \cap H_{m s}, \quad|a| \leqq|b|$, so bezeichnen wir mit $V(a, b)$ die Menge

$\left\{x \in R^{n} \mid \cos \left(x-a, e^{1}\right)>\cos \alpha\right\} \cap\left\{x \in R^{n} \mid \cos \left(x-b, e^{1}\right)<-\cos x\right\}$.

Im folgenden wird $V(a, b)$ das von der Strecke $\{x \in J|| a|\leqq| x|\leqq| b \mid\}$ bestimmte Kegelelement genannt. Sei $p$ so gross, dass die abgeschlossene Hülle des Zylinders $Z\left(\frac{1}{2 p} \tan \alpha\right)$ in $G$ liegt. Für das Volumenmass von $Z$ gilt

$$
m\left(Z\left(\frac{1}{2 p} \tan \alpha\right)\right)=\left(\frac{1}{2 p} \tan \alpha\right)^{n-1} \Omega_{n-1} .
$$

Nach der Definition von $H_{m s}$ enthält $V^{\prime}(a, b)$ eine Kugel $K_{r}(y)$, 
deren Mittelpunkt $y$ auf $J_{k}^{\prime}$ derart liegt, dass $|y-f(a)|=|y-f(b)|$ ist, und deren Radius $r$ die Beziehung

$$
r \geqq \frac{\alpha}{s} \frac{|f(a)-f(b)|}{2}
$$

erfüllt. Daher gilt

$$
\Omega_{n}\left(\frac{\alpha}{2 s}|f(a)-f(b)|\right)^{n} \leqq \Omega_{n} r^{n} \leqq m\left(V^{\prime}(a, b)\right) \leqq m\left(V_{k}^{\prime}\right),
$$

worin $V_{k}$ das von der Strecke $J_{k}$ bestimmte Kegelelement ist. Für den Durchmesser $d_{k}$ von $J_{k}^{\prime} \cap H_{m s}^{\prime}$ erhält man hieraus die Abschätzung

$$
d_{k}^{n}=\sup |f(a)-f(b)|^{n} \leqq \frac{2^{n}}{\Omega_{n}}(s / \alpha)^{n} m\left(V_{k}^{\prime}\right)
$$

Es sei $\delta(p)=\max \left(d_{1}, \ldots, d_{N}\right)$. Da $\left(\bigcup_{1}^{N} J_{k}^{\prime}\right) \cap H_{m s}^{\prime}$ eine Überdeckung von $\left(\bigcup_{1}^{M} O_{i}^{\prime}\right) \cap H_{m s}^{\prime}$ ist, erhält man aus (3.5), (3.6), (3.7) und aus der Hölderschen Ungleichung

$$
\begin{aligned}
& A\left(\left(\bigcup_{1}^{M} O_{i}^{\prime}\right) \cap H_{m s}^{\prime}, \quad \delta(p)\right)^{n} \leqq\left(\sum_{1}^{N} d_{k}\right)^{n} \\
& \leqq N^{n-1} \sum_{1}^{N} d_{k}^{n} \leqq \frac{2^{n}}{\Omega_{n}}(s / a)^{n} N^{n-1} \sum_{1}^{N} m\left(V_{k}^{\prime}\right) \\
& <\frac{2^{n}}{\Omega_{n}}(s / \alpha)^{n} m\left(Z^{\prime}\left(\frac{1}{2 p} \tan \alpha\right)\right) \cdot p^{n-1}\left(\sum_{1}^{M} \Lambda\left(O_{i}\right)+\frac{2 M}{p}\right)^{n-1} \\
& <\frac{2^{n}}{\Omega_{n}} \tan ^{n-1} \alpha \Omega_{n-1}(s / \alpha)^{n} \frac{m\left(Z^{\prime}\left(\frac{1}{2 p} \tan \alpha\right)\right)}{m\left(Z\left(\frac{1}{2 p} \tan \alpha\right)\right)}\left(\Lambda(O)+\frac{2 M}{p}\right)^{n-1}
\end{aligned}
$$

Wir lassen nun $p$ gegen unendlich streben. Wegen der Stetigkeit von $f$ ist $\lim \delta(p)=0$, und durch eine einfache Betrachtung ergibt sich $p \rightarrow \infty$

$$
\Lambda^{*}\left(\left(\bigcup_{1}^{M} O_{i}^{\prime}\right) \cap H_{m s}^{\prime}\right) \leqq \varkappa \frac{s}{\alpha} \sqrt[n]{\sigma(0)(\Lambda(0))^{n-1}}
$$

Dies gilt für jedes $M>0$, woraus nach (3.4) folgt

$$
\Lambda^{*}\left(E^{\prime} \cap H_{m s}^{\prime}\right) \leqq \varkappa \frac{s}{\alpha} \sqrt[n]{\sigma(0)(\Lambda(0))^{n-1}}
$$


Da $O$ eine beliebige offene Überdeckung von $E \cap H_{m s}$ ist, schliesst man hieraus die Gültigkeit von (3.3).

Aus der Abschätzung (3.2) und der Bedingung $1^{\circ}$ folgt nun, dass $f: J \rightarrow J^{\prime}$ nullmengentreu ist. Ist nämlich $\Lambda(E)=0$, so gilt $\Lambda^{*}\left(E^{\prime} \cap H_{s}^{\prime}\right)=0$ für jedes $s>0$ und daher $A^{*}\left(E^{\prime}\right)=\lim A^{*}\left(E^{\prime} \cap H_{s}^{\prime}\right)=0$.

Für jede messbare Menge $E \subset J$ ist $E^{\prime}$ also messbar, und der Bedingung (3.1) gemäss gilt für jedes $s>K$

$$
\Lambda\left(E^{\prime}\right)=\Lambda\left(E^{\prime} \cap H_{s}^{\prime}\right) \leqq \varkappa \frac{s}{\alpha} \sqrt[n]{\sigma(0)(\Lambda(E))^{n-1}} .
$$

Daher ist

$$
\Lambda\left(E^{\prime}\right) \leqq x \frac{K}{\alpha} \sqrt[n]{\sigma(0)(\Lambda(E))^{n-1}},
$$

woraus die absolute Stetigkeit von $f$ folgt.

Zweitens beweist man die lokale $L^{n}$-Integrierbarkeit der partiellen Ableitungen von $f$.

Lemma 2. Unter den Voraussetzungen von Lemma 1 ist $f A S G_{n}$ in $G$.

Beweis. Wegen der Symmetrie wird die Behauptung bewiesen, wenn wir zeigen, dass die Ableitung $\partial_{1} f$ lokal $L^{n}$-integrierbar ist. Man bezeichne mit $K_{m s}$ die Menge aller Punkte $x \in G$, für die $\omega_{m}\left(x, e^{1}, \alpha\right) \geqq \alpha / s$ ist. Ferner sei $E$ die Menge derjenigen Punkte von $G$, wo die endliche Ableitung $\partial_{1} f$ existiert; nach der $A S G$-Eigenschaft von $f$ und gemäss dem Satz von Fubini ist $m(G-E)=0$.

Es sei $x^{0} \in E \cap K_{m s}$ ein $x_{1}$-Dichtepunkt von $E \cap K_{m s} \quad$ (vgl. z.B. Lehto - Virtanen [6], S. 120). Wir können dann eine Folge $t_{1}, t_{2}, \ldots$ von verschiedenen reellen Zahlen mit den Eigenschaften $0<t_{v}<1 / m, \lim t_{\nu}=$ 0 , derart wählen, dass die Punkte $x^{v}=x^{0}+t_{v} e^{1}$ in $K_{m s}$ liegen. Für genügend grosse Werte von $v$ enthält $D$ das Kegelelement $V\left(x^{0}, x^{v}\right)$. In $V^{\prime}\left(x^{0}, x^{v}\right)$ können wir wieder eine Kugel konstruieren, deren Radius $r\left(x^{0}, x^{v}\right)$ $\geqq \frac{\alpha}{2 s}\left|f\left(x^{v}\right)-f\left(x^{0}\right)\right|$ ist, vgl. S. 8. Da $\left|x^{v}-x^{0}\right|=\frac{2^{n-1} n}{\Omega_{n-1} \tan ^{n-1} \alpha} m\left(V\left(x^{0}, x^{v}\right)\right.$ ist, erhält man hieraus die Abschätzung

$$
\begin{aligned}
& \Omega_{n} \frac{\left|f\left(x^{v}\right)-f\left(x^{0}\right)\right|^{n}}{\left|x_{1}^{v}-x_{1}^{0}\right|^{n}} \leqq 2^{n} \Omega_{n}(s / \alpha)^{n} \frac{\left(r\left(x^{0}, x^{v}\right)\right)^{n}}{\left|x^{v}-x^{0}\right|^{n}} \\
& \leqq \frac{2 \Omega_{n-1} \tan ^{n-1} \alpha}{n}(s / \alpha)^{n} \frac{m\left(V^{\prime}\left(x^{0}, x^{\nu}\right)\right.}{m\left(V\left(x^{0}, x^{v}\right)\right.} .
\end{aligned}
$$


Setzt man $\tau(A)=m(f(A))$ für jede Borelsche Menge $A \subset G$, so existiert die endliche Ableitung $\tau^{\prime}(x)$ für fast alle $x \in G$. Wir nehmen an, dass der obige Punkt $x^{0}$ auch diese Bedingung erfüllt. Da $\partial_{1} f\left(x^{0}\right)$ existiert, folgt aus (3.8)

$$
\left|\partial_{1} f\left(x^{0}\right)\right|^{n} \leqq \frac{x^{n}}{n}(s / \alpha)^{n} \tau^{\prime}\left(x^{0}\right)
$$

wo $x$ die Konstante in (3.2) ist.

Fast alle Punkte von $E \cap K_{m s}$ sind $x_{1}$-Dichtepunkte, und daher ergibt sich durch Integration

$$
\int_{C \cap K_{m s}}\left|\partial_{1} f\right|^{n} \leqq \frac{x^{n}}{n}(s / \alpha)^{n} \int_{C \cap K_{m s}} \tau^{\prime} \leqq \frac{x^{n}}{n}(s / \alpha)^{n} m\left(C^{\prime}\right),
$$

wo $C$ eine beliebige kompakte Teilmenge von $G$ ist. Bezeichnet man mit $K_{s}$ die Vereinigungsmenge $\bigcup_{m=1}^{\infty} K_{m s}$, so gilt ferner

$$
\int_{C \cap K_{s}}\left|\partial_{1} f\right|^{n} \leqq \frac{\varkappa^{n}}{n}(s / \alpha)^{n} m\left(C^{\prime}\right) .
$$

Da $K_{s}$ alle Punkte $x \in G$ enthält, für die $\omega\left(x, e^{1}, \alpha\right)>\alpha / s$ ist, gilt die Gleichheit $m\left(C \cap K_{s}\right)=m(C)$ für jedes $s>K$. Hieraus ergibt sich

$$
\int_{\dot{C}} \mid \partial_{1} f_{\mid}^{n} \leqq \frac{\varkappa^{n}}{n}(s / x)^{n} m\left(C^{\prime}\right)
$$

und damit ist Lemma 2 bewiesen.

Als Abschluss des Beweises von Satz 1 ist noch zu zeigen, dass $f$ die Doppelungleichung

$$
L(x, f)^{n}\left|K^{n-1} \leqq\right| J(x, f) \mid \leqq K^{n-1} l(x, f)^{n}
$$

fast überall in $G$ befriedigt. Als Vorbereitung betrachten wir die Veränderung eines gewöhnlichen Winkels bei einer linearen Abbildung.

Lemma 3. Es sei $A, A(x)=\sum_{i=1}^{n} \lambda_{i} x_{i} e^{i}, \lambda_{1} \geqq \ldots \geqq \lambda_{n}>0$, eine lineare Abbildung von $R^{n}$ und $0<\alpha<\pi / 2$. Für jeden Winkel $(x, y)=\alpha$ gilt dann

$$
\beta \geqq 2 \arctan \left(\frac{\lambda_{n}}{\lambda_{1}} \tan \frac{\alpha}{2}\right),
$$

wo $\beta$ der Bildwinkel von $(x, y)$ ist. Die Schranke ist die bestmögliche. 
Beweis. Betrachtet man die Einschränkung von $A$ auf die $x y$-Ebene, so sieht man durch elementare Betrachtungen, dass $\beta$ am kleinsten ist, wenn $x$ und $y$ in der $x_{1} x_{n}$-Ebene in bezug auf die $x_{1}$-Achse symmetrisch liegen. In diesem Fall gilt auch die Gleichheit in (3.11).

Da $f$ nach Lemma 2 fast überall in $G$ differenzierbar ist (vgl. z.B. Väisälä [10], Lemma 3), folgt die Behauptung von Satz 1 schliesslich aus dem folgenden Hilfssatz.

Lemma 4. Es sei $x$ ein Punkt von $G$, in dem $f$ differenzierbar ist. Ist

$$
\operatorname{arc} \sin \omega(x, v, \alpha) \geqq \alpha / K
$$

für jedes $v$ und für alle Winkel $0<\alpha<\pi / 2$, so gilt (3.10), wo die Schranke $K^{n-1}$ die bestmögliche ist.

Beweis. Ist $L(x, f)=0$, so gilt (3.10) trivialerweise. Andernfalls erreicht man durch geeignete Ähnlichkeitstransformationen, dass $x=0$ und die Koordinatenfunktionen $g_{i}$ von $g=D f(0)$ die einfache Form $g_{i}(x)=\lambda_{i} x_{i} \quad$ haben, wo $\lambda_{1} \geqq \lambda_{2} \geqq \ldots \geqq \lambda_{n} \geqq 0$ die Halbachsen der Bildellipsoide von $|x|=1$ sind. Es ist leicht ersichtlich, dass $\lambda_{n}>0$ ist und dass die Bedingung (3.12) auch für die Abbildung $g$ gilt.

Nach Lemma 3 und der Bedingung (3.12) ist $\min _{v} \operatorname{arc} \sin \omega(0, v, \alpha)=$ $2 \arctan \left(\frac{\lambda_{n}}{\lambda_{1}} \tan \frac{\alpha}{2}\right) \geqq \alpha / K$ für jedes $0<\alpha<\pi / 2$. Durch den Grenzübergang $\alpha \rightarrow 0$ erhält man hieraus die genaue Abschätzung $\lambda_{1} / \lambda_{n} \leqq K$. Die Doppelgleichung (3.10) ist dann gültig, und durch die spezielle Wahl $\lambda_{1}=\ldots=\lambda_{n-1}=K, \quad \lambda_{n}=1$ oder $\lambda_{1}=K, \lambda_{2}=\ldots=\lambda_{n}=1$ sieht man, dass die Konstante $K^{n-1}$ die kleinstmögliche ist.

\section{§ 4. Verzerrung eines Raumwinkels bei der Quasikonformität}

Bei einer $K$-quasikonformen Abbildung ebener Gebiete kann die genaue Schranke für die Winkelverzerrung nach unten in allen Punkten hergeleitet werden, vgl. [2] und [8]. Im Falle $n \geqq 3$ ist die Situation in allen regulären Punkten gleich; wegen des speziellen Charakters der räumlichen Dilatation ist das Resultat hierbei jedoch nicht den Formeln (3.10) und (3.12) ähnlich. In einem nicht-regulären Punkt der Abbildung scheint die Bestimmung der genauen Schranke dagegen sehr schwierig zu sein, und wir begnügen uns damit, nur eine positive von $K$ und $\alpha$ abhängige Schranke zu ermitteln (Satz 3). 
Satz 2. Es sei $f: G \rightarrow G^{\prime}$ eine $K$-quasikonforme Abbildung in $R^{n}$. Für fast alle Punkte $x \in G$ gilt dann

$$
\arcsin \omega(x, v, \alpha) \geqq 2 \arctan \left(K^{-2 / n} \tan (\alpha / 2)\right),
$$

wo $0<\alpha<\pi / 2$ ist. Die Schranke ist die bestmögliche.

Beweis. Nach Väisälä [9], Theorem 6.10 und 6.13, ist $f$ differenzierbar und $J(x, f) \neq 0$ für fast jedes $x \in G$. Wir können annehmen, dass $x=0$ ein solcher Punkt ist und dass die Ableitung $g=D f(0)$ durch

$$
g(x)=\sum_{i=1}^{n} \lambda_{i} x_{i} e^{i}, \quad \lambda_{1} \geqq \ldots \geqq \lambda_{n}>0,
$$

gegeben ist. Die Formel (4.1) folgt nun fast unmittelbar aus (3.11) wegen der Ungleichung $\lambda_{n} / \lambda_{1} \geqq K^{-2 / n}$, vgl. (1.2).

Ferner ist die Genauigkeit von (4.1) zu begründen. Da (3.11) scharf ist, genügt es hierfür, eine solche $K$-quasikonforme Abbildung $g(x)=\sum_{i=1}^{n} \lambda_{i} x_{i} e^{i}$ zu konstruieren, für die $\lambda_{1} / \lambda_{n}=K^{2 / n}$ ist. $Z u$ diesem $Z$ weck setze man

$$
\lambda_{1}=K^{\frac{2}{n}}, \quad \lambda_{2}=K^{\frac{2}{n}-d}, \lambda_{3}=K^{\frac{2}{n}-2 d}, \ldots, \lambda_{n}=1
$$

mit $d=2 / n(n-1)$. Da die Summe der Exponenten gleich eins ist, gilt die Doppelgleichung

$$
\frac{1}{K} \hat{\lambda}_{1}^{n}=\lambda_{1} \ldots \hat{\lambda}_{n}=K \hat{\lambda}_{n}^{n},
$$

und $g$ hat also die gewünschten Eigenschaften.

Wir gehen jetzt zu dem allgemeinen Fall über und benutzen hierfür das folgende Resultat von Gehring (vgl. [5], Theorem 11): Ist $f: D \rightarrow D^{\prime}$, $\partial D \neq \varnothing, \partial D^{\prime} \neq \varnothing$, eine $K$-quasikonforme Abbildung, so gilt

$$
\frac{|f(x)-f(y)|}{\varrho\left(f(x), \partial D^{\prime}\right)} \leqq \Theta_{K}\left(\frac{|x-y|}{\varrho(x, \partial D)}\right)
$$

für alle $x, y \in D$ mit $|x-y|<\varrho(x, \partial D)$, wo $\varrho(x, \partial D)$ und $\varrho\left(f(x), \partial D^{\prime}\right)$ die Entfernungen der Punkte $x$ bzw. $f(x)$ vom Rande $\partial D$ bzw. $\partial D^{\prime}$ sind. Hier ist $\Theta_{K}$ eine bestimmte, auf dem Intervall $(0,1)$ definierte, echt zunehmende und stetige reellwertige Funktion, die in den Endpunkten von $(0,1)$ die Grenzwerte $\lim _{t \rightarrow 0} \Theta_{K}(t)=0, \lim _{t \rightarrow 0} \Theta_{K}(t)=\infty$ besitzt.1)

1) Dieses Resultat ist von Caraman [3] auf den $n$-dimensionalen Fall verallgemeinert worden. 
Mit Hilfe dieser Formel beweisen wir den folgenden Satz, wobei die entsprechende Verzerrungsfunktion im Falle der $n$-dimensionalen Abbildungen mit $\Theta_{K}^{n}$ bezeichnet wird.

Satz 3. Es sei $f: G \rightarrow G^{\prime}$ eine $K$-quasikonfore Abbildung in $R^{n}$. In allen Punkten gilt dann für jedes $0<\alpha<\pi / 2$ und $|v|=1$ die $A b$ schätzung

$$
\omega(x, v, \alpha) \geqq\left(\Theta_{K}^{n}\right)^{-1}(\sin \alpha),
$$

wo $\left(\Theta_{K}^{n}\right)^{-1}$ die Umkehrung von $\Theta_{K}^{n}$ ist.

Beweis. Man nehme $x=f(x)=0$ an. Es genügt offenbar, den Kegel $W^{+}\left(0, e^{1}, \alpha\right)=W\left(0, e^{1}, \alpha\right) \cap\left\{x \in R^{n} \mid x_{1}>0\right\} \quad$ zu betrachten. Wir wählen einen Punkt $x$ der positiven $x_{1}$-Achse derart, dass die Kugel $K=$ $\left\{z \in R^{n}|| z-f(x)|<| f(x) \mid\right\}$ in $G^{\prime}$ liegt. Für beliebiges $y \in W^{+}\left(0, e^{1}, \alpha\right)$ schätzen wir das Verhältnis $|f(x)-f(y)| /|f(y)|$ nach unten ab. Liegt $f(y)$ in $K$, so liefert der obige Verzerrungssatz von Gehring, auf die Abbildung $f^{-1} \mid K$ angewandt,

$$
\sin \alpha \leqq \frac{|x-y|}{|x|} \leqq \Theta_{K}^{n}\left(\frac{|f(x)-f(y)|}{|f(x)|}\right) .
$$

Da andernfalls $|f(x)-f(y)| / \mid f(x) \geqq 1$ ist, schliesst man hieraus die Gültigkeit der Formel (4.2).

Aus den obigen Resultaten erhält man unmittelbar die folgenden notwendigen und hinreichenden Bedingungen für die Quasikonformität.

Satz 4. Ein Homöomorphismus $f: G \rightarrow G^{\prime}$ zwischen den Gebieten $G$ und $G^{\prime}$ von $R^{n}$ ist quasikonform genau dann, wenn

$$
\begin{aligned}
& 1^{\circ} . \omega(x, v, \alpha)>0 \text { für jedes } x \in G,|v|=1,0<\alpha<\pi / 2, \\
& 2^{\circ} . \quad \operatorname{essinf}_{x \in G}\left(\inf _{\substack{0<\alpha<\pi / 2 \\
|v|=1}} \frac{\omega(x, v, \alpha)}{\alpha}\right)>0 .
\end{aligned}
$$

\section{§ 5. Winkelbegriff von Agard-Gehring}

In seiner Arbeit [1] hat Agard einen topologischen Winkel definiert, der in engem Zusammenhang mit unserem steht. Der Bildwinkel des erzeugenden Winkels von $W^{+}(x, v, \alpha)=W(x, v, \alpha) \cap\left\{y \in R^{n} \mid(y-x, v) \leqq \pi / 2\right\}$ bei einem Homöomorphismus $f$ wird von Agard durch den Ausdruck 
(5.1) $\quad A^{\prime}(x, v, \alpha)=2 \operatorname{arc} \sin \left(\liminf _{y, z \rightarrow x} \frac{|f(y)-f(z)|}{|f(y)-f(x)|+|f(z)-f(x)|}\right)$

definiert, in dem $y$ auf der Kegelfläche $W^{+}(x, v, \alpha)$ und $z$ auf der Achse $L^{+}(x, v, \alpha)$ von $W^{+}(x, v, \alpha)$ liegen.

Man setze nun

$$
A(x, v, \alpha)=\operatorname{Min}\left(A^{\prime}(x, v, \alpha), A(x,-v, \alpha)\right) .
$$

Obgleich die Winkel $A(x, v, \alpha)$ und arc $\sin \omega(x, v, \alpha)$ im allgemeinen nicht gleich gross sind, ist ihr Verhältnis doch beschränkt, wie wir im Folgenden zeigen werden. Da $A(x, v, \alpha)$ dazu in allen regulären Punkten von $f$ mit arc $\sin \omega(x, v, \alpha)$ übereinstimmt, sind Satz 4 und das entsprechende Resultat von Agard unmittelbare gegenseitige Folgerungen.

Satz 5. Es sei $f: G \rightarrow G^{\prime}$ ein Homöomorphismus zwischen den Gebieten $G \subset R^{n}$ und $G^{\prime} \subset R^{n}$ sowie $0<\alpha<\pi / 2$ ein fester Winkel. Für jedes $v$ gilt dann die Doppelungleichung

(5.2) $2 \arcsin \left(\min \left(\frac{\omega(x, v, \alpha)}{4}, \frac{1}{2}\right)\right) \leqq A(x, v, \alpha) \leqq 2 \arcsin \omega(x, v, \alpha)$.

Beweis. Wir betrachten die Punkte $y \in W^{+}(x, v, \alpha)$ und $z \in L^{+}(x, v, \alpha)$. Die rechte Ungleichung ergibt sich unmittelbar aus der Abschätzung

$$
\frac{|f(y)-f(z)|}{|f(x)-f(y)|+|f(x)-f(z)|} \leqq \frac{|f(y)-f(z)|}{|f(x)-f(z)|} .
$$

Zur Begründung der linken Ungleichung (5.2) bemerken wir zuerst, dass

$$
\frac{|f(y)-f(z)|}{|f(y)-f(x)|+|f(z)-f(x)|}>\frac{|f(y)-f(z)|}{4|f(z)-f(x)|}
$$

für jedes $y$ mit $|f(y)-f(x)|<3|f(x)-f(z)|$ gilt. Andernfalls erhält man durch eine einfache elementargeometrische Betrachtung die Ungleichung

$$
\frac{|f(y)-f(z)|}{|f(y)-f(x)|+|f(z)-f(x)|} \geqq \frac{1}{2} .
$$

Die linke Ungleichung (5.2) folgt fast unmittelbar aus (5.3) und (5.4).

Aus dem folgenden Beispiel geht schliesslich hervor, dass arc sin $\omega(x, v, \alpha) \neq A(x, v, \alpha)$ schon im Falle $n=2$ sein kann.

Man betrachte für ein festes $a>0$ in der komplexen Ebene die Bogen 